\title{
Spectral characteristics of turbulent boundary layers - comparison of Particle Image Velocimetry and Thermal Anemometry
}

\author{
Klára Jurčáková ${ }^{1, *}$, Radka Kellnerová ${ }^{1}$, Pavel Procházka ${ }^{1}$ and Pavel Antoš ${ }^{1}$ \\ ${ }^{1}$ Institute of Thermomechanics, Czech Academy of Sciences, Dolejškova 5, 182 00, Praha, Czech Republic
}

\begin{abstract}
Flow in turbulent boundary layers over various rough surfaces was measured by the timeresolved particle image velocimetry and the thermal anemometry. The experimental methods showed very good agreement in both mean and spectral characteristics taking into account each method limitations. The spectral characteristics supported the attached eddy hypothesis. The most energetic structures were growing with the wall-normal distance and they achieved sizes equal to 3 to 8 boundary layer depths.
\end{abstract}

\section{Introduction}

The nature of wall-bounded turbulence over a rough wall is important question, because its understanding is critical for prediction of many engineering and environmental flows. In the turbulent boundary layer (BL) over a rough surface, the lowest parts (the inner layer where the viscous forces are dominant, the buffer layer and lower part of the inertial sublayer) are replaced by the roughness sublayer, where the flow is dependent on the position of the roughness elements. The flow in the inertial layer and the outer layer is influenced only by an integral roughness forcing [1]. In this region, the turbulence spectrum is classically divided into a low wavenumber range where motions scale with $\mathrm{BL}$ depth $\delta$, an intermediate wavenumber range where motions scale with wall-normal distance $z$, and a high wavenumber range where motions scale with the Kolmogorov dissipation length $\eta$ [2].

A particular question is the behaviour of the turbulence spectrum at different Reynolds numbers and for the different surface roughness. Jimenez in [3] did an extensive review on the magnitude of the most energetic length scales in BL over rough surfaces. The scale of structures supposed to grow with the roughness but the sources are not consistent in magnitude. This study is a contribution to this discussion.

\section{Experimental setup}

Boundary layers developed over 4 different rough surfaces were investigated in the blow-type wind tunnel with dimensions: $250 \mathrm{~mm}$ x $250 \mathrm{~mm} \times 3000 \mathrm{~mm}$ (width $\mathrm{x}$ height $\mathrm{x}$ length). The wind-tunnel floor was covered by surface roughness consisted of erected metal plates of a uniform height arranged in staggered arrays. Four different heights $k(2 ; 4 ; 8 ; 16) \mathrm{mm}$ of the roughness elements were used for four setups labelled M2, M3, M4, and M5, respectively (label M1 was reserved for a flat surface). The roughness spacing (the roughness width of $12.5 \mathrm{~mm}$, the lateral spacing of $25 \mathrm{~mm}$ and the stream-wise spacing of $42 \mathrm{~mm}$ ) was identical for all setups.

Flow fields were measured by two independent methods. The first was 2D time-resolved Particle Image Velocimetry (TR PIV) consisted of diode pumped $\mathrm{Nd}$ :YLF laser (10 $\mathrm{mJ}$ in a pulse), one camera with resolution $1280 \times 800$ pixels, interrogation area size $32 \times$ 32 pixels with $50 \%$ overlapping, field of view $140 \mathrm{~mm} \mathrm{x}$ $90 \mathrm{~mm}$, spatial resolution $1.8 \mathrm{~mm}$ ( 77 x 49 vectors). The TR-PIV system run in two modes: the dynamic mode had sampling frequency $1000 \mathrm{~Hz}$ and sampling time $4 \mathrm{~s}$ (labelled PIVD); the statistic mode had sampling frequency $100 \mathrm{~Hz}$ and sampling time $40 \mathrm{~s}$ (labelled PIVS). 4000 time steps were collected in both modes in 1 run. The centre of the vertical measurement plane was located $1824 \mathrm{~mm}$ downstream from the wind tunnel

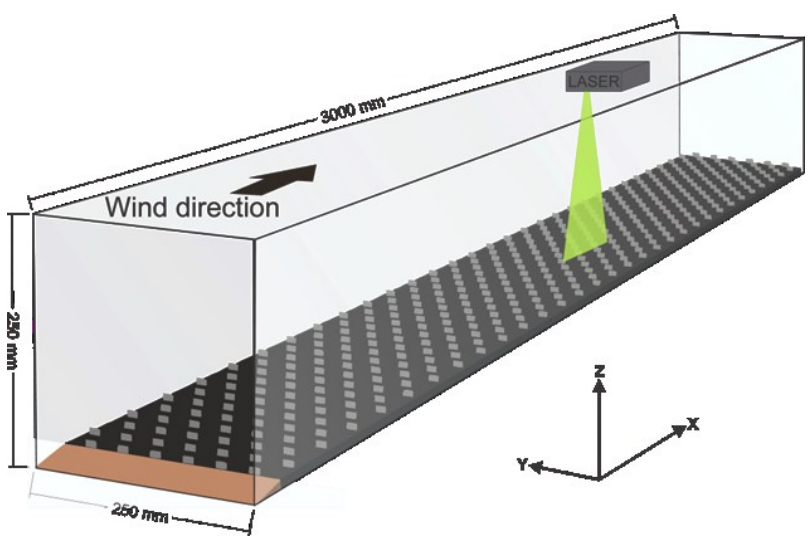

Fig. 1. Wind tunnel with roughness elements (setup M4) and position of the laser sheet.

\footnotetext{
* Corresponding author: klara.jurcakova $@$ it.cas.cz
} 
entrance, see Fig.1. The $x$-axis was parallel with the mean flow direction, $y$-axis was laterally oriented and $z$ axis was vertical. The respective velocity components are depicted $U, V$ and $W$.

The second method was 2-D constant temperature anemometry (HW) with X-array wire probe (Dantec 55P51). The wires have a $5 \mu \mathrm{m}$ diameter, they are $3 \mathrm{~mm}$ long and the separation distance is $0.6 \mathrm{~mm}$. Vertical profiles were sampled at the centre of the PIV measurement plane $(x=1824 \mathrm{~mm})$. The sampling frequency was $25 \mathrm{kHz}$ and sampling time was $20 \mathrm{~s}$.

\section{Mean flow characteristics}

The flow field was measured for various free-stream wind speeds for all setups and both measurement methods. Seven investigated free-stream velocities was in the range from $5 \mathrm{~m} / \mathrm{s}$ to $23 \mathrm{~m} / \mathrm{s}$ which corresponds to Reynolds number Re from 18000 to 180000 (based on the boundary layer depth $\delta$ and the free stream velocity $U_{\delta}$ ), or $R e^{*}$ from 1 to 276 (based on roughness length $z_{0}$ and friction velocity $\left.u^{*}\right)$, or $R e_{\tau}$ from $750-14500$ (based on $\delta$ and $u^{*}$ ).

The vertical profiles of the mean wind speed $U$, the stream-wise velocity fluctuations $\sigma_{u}$, the wall-normal velocity fluctuations $\sigma_{w}$, and the turbulent shear stress $u$ ' $w$ ' of the naturally developed boundary layers at the measurement position $\mathrm{x}=1824 \mathrm{~mm}$ at the wind-tunnel centreline are shown in Fig. 2. There is a very good agreement between dimensionless vertical profiles measured by either different methods or at different Reynolds numbers except about 30\% underestimation of $u$ 'w' values measured by HW. This offset was caused by the limitation of the $\mathrm{X}$ probe measurement in the highly turbulent flows with turbulence intensities higher than $20 \%$.

All profiles in Fig. 2 are showing a step change just below the height $0.2 \delta$. This is the height of the roughness sublayer, where the flow is influenced by the individual roughness elements. Above this height, the flow is influenced by an integral roughness force and it becomes homogeneous in lateral and stream-wise direction in statistical sense. We will exclude the surface sublayer in the following discussions.

Table 1. Properties of the boundary layers.

\begin{tabular}{l|cccc}
\hline & M2 & M3 & M4 & M5 \\
\hline$\delta[\mathrm{mm}]$ & 53 & 74 & 89 & 117 \\
$\delta / k$ & 35 & 15 & 11 & 7 \\
$u^{*} / U_{\delta}[\%]$ & 4.4 & 5.9 & 6.3 & 7.9 \\
$z_{0}[\mathrm{~mm}]$ & 0.04 & 0.47 & 0.8 & 2.3 \\
$\delta *[\mathrm{~mm}]$ & 9.8 & 18.2 & 22.9 & 35.1 \\
$\theta^{*}[\mathrm{~mm}]$ & 6.7 & 10.5 & 12.8 & 16.7 \\
$H=\delta^{*} / \theta^{*}$ & 1.5 & 1.7 & 1.8 & 2.1 \\
\hline
\end{tabular}

The mean wind speed profile can be characterized by the parameters given in Table 1 . The values are averages of data for all the wind speeds and obtained by both TR-PIV and HW measurement. Boundary layer depth $\delta$ was set as a height at which the local mean wind speed reached $99 \%$ of the maximal velocity. The free stream velocity $U_{\delta}$ is mean velocity at the $\delta$ height. The $\mathrm{BL}$ depth is increasing with the increasing height of the roughness but not proportionally, therefore the ratio of $\mathrm{BL}$ which is occupied by the roughness elements is increasing (from $1 / 35$ for the M2 setup to $1 / 7$ to M5 setup). The friction velocity $u^{*}$ and the roughness height $z_{0}$ was obtained by the least-square fit of the logarithmic law defined as

$$
U=\frac{u^{*}}{\kappa} \ln \frac{z}{z_{0}} .
$$
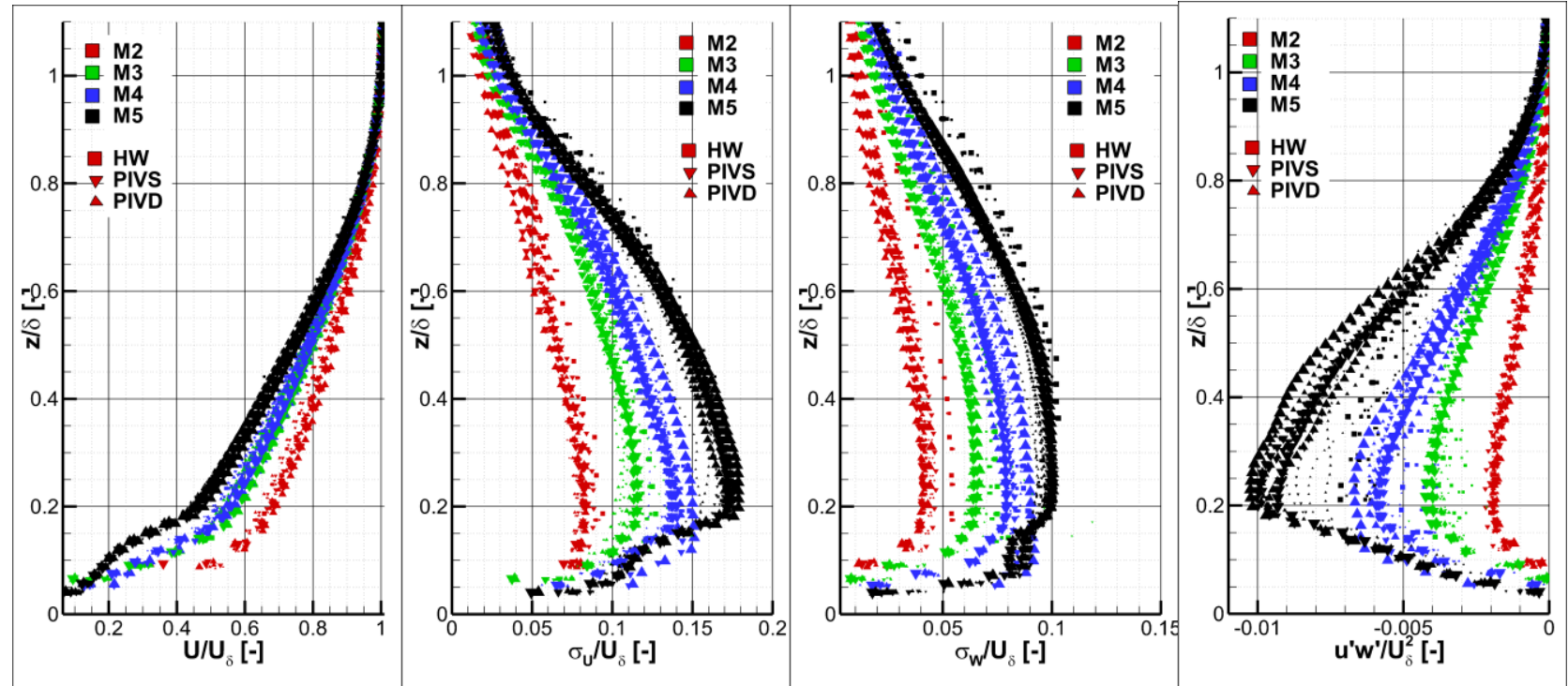

Fig. 2. Vertical profiles of mean wind speed $U$, stream-wise velocity fluctuations $\sigma_{u}$, wall-normal velocity fluctuations $\sigma_{u}$, and turbulent shear stress $u^{\prime} w^{\prime}$. Symbol's colour gives the setup, symbol's shape gives the measurement method, and symbol's size is given by the free stream velocity. 

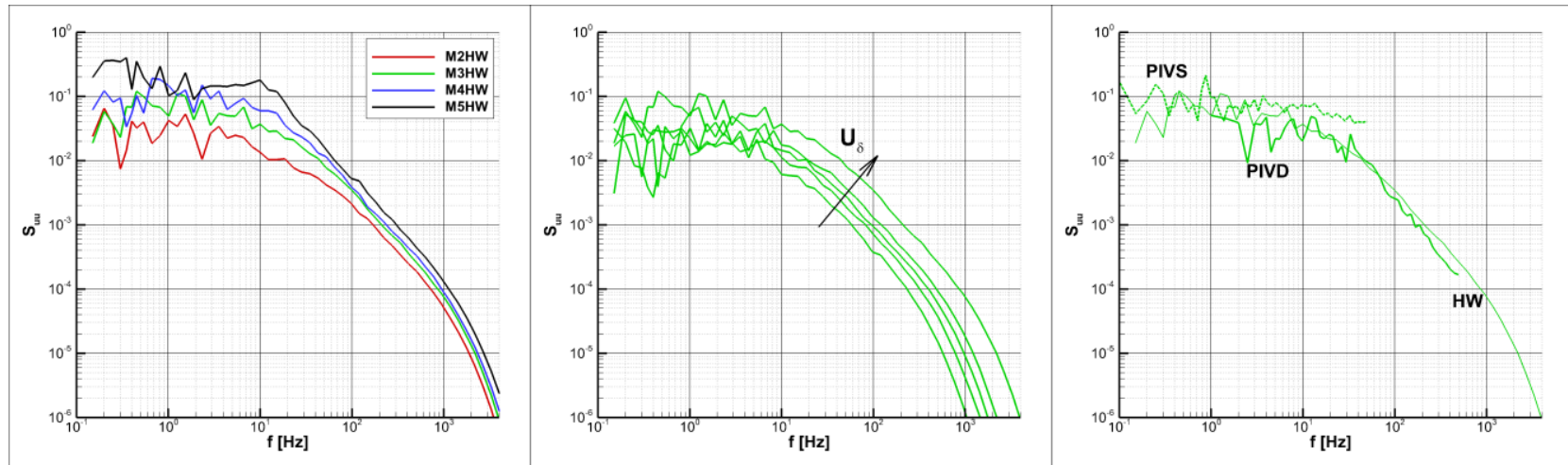

Fig. 3. Dependence of the power spectral density on the surface roughness, HW data, $U_{\delta}=10 \mathrm{~m} / \mathrm{s}, \mathrm{z} / \delta=0.33$ (left); dependence on the free stream velocity, HW data, setup M3, $z / \delta=0.33$ (middle), comparison of the spectral curves for HW, PIVS and PIVD methods setup M3, $U_{\delta}=10 \mathrm{~m} / \mathrm{s}, z / \delta=0.33$ (left).

The ratio of the displacement thickness $\delta^{*}$ and the momentum thickness $\theta^{*}$ is known as a shape factor $H$. The typical value of the shape factor for turbulent $\mathrm{BL}$ over a smooth wall is 1.3-1.4. The higher values are associated with the adverse pressure gradient or the presence of roughness [4].

\section{Energetic spectra}

The power spectra densities (PSD) $S_{u u}$ and $S_{w w}$ were obtained as fast Fourier transform (FFT) of discrete measured velocity fluctuation in stream-wise and wallnormal direction data $\left(u^{\prime}, w^{\prime}\right)$ according to

$$
\begin{aligned}
& S_{u u}=2 * \operatorname{abs}\left(\frac{F F T\left(u^{\prime}\right)}{N}\right)^{2} / N * S T, \\
& S_{w w}=2 * \operatorname{abs}\left(\frac{F F T\left(w^{\prime}\right)}{N}\right)^{2} / N * S .
\end{aligned}
$$

where $N$ is the number of samples and $S T$ is the sampling time.

The limitations for resolved frequencies are given by the sampling frequency, the sampling time $S T$ and the resolution of the instrument for the given measurement. The maximal frequency which can be resolved is Nyquist frequency $f_{N Y}$ and it is equal half of the sampling frequency. The maximal resolved frequency due to the spatial resolution of the methods $f_{S R}$ is given as a ratio of the local mean velocity $U$ and the method spatial limit (HW: distance of the wires $0.6 \mathrm{~mm}$, PIV: interrogation area size $1.8 \mathrm{~mm}$ ). The minimal resolved frequency $f_{S T}$ is the inverse sampling time. The maximal resolved frequency for a given point is $\min \left(f_{N Y}, f_{S R}\right)$. The minimal frequency with physical meaning is approx. $f_{S T} * 10$ (at least 10 repetitions of an event). Summary of frequency limits is given in Table 2. The actual values of maximal frequencies depend on the local mean velocity $U$. For example, if $U=5 \mathrm{~m} / \mathrm{s}, \mathrm{HW}: f_{S R}=8300 \mathrm{~Hz}, \mathrm{PIV}: f_{S R}=2700$ Hz. Nyquist frequency was the limit in majority of PIV spectra, while $f_{S R}$ based on the separation of the wires was the limiting factor for all HW spectra.

Power spectra densities $S_{u u}$ and $S_{w w}$ are showing content of turbulent kinetic energy (TKE) in a given component and a given frequency interval $f+\Delta f$, where $\Delta f=f_{S T}$. The absolute value of TKE is increasing with the higher roughness as well as with the higher free stream velocity and therefore PSD is increasing likewise (examples given in Fig. 3, left and middle). The HW data covered at least 4 decades of frequency values, while the both of the PIV dataset had limited frequency range. Example of the methods' comparison is given in Fig. 3, right. The agreement is good in the inner part of resolved intervals, while the upper and lower tails were suffering by high frequency aliasing and lack of the statistical representativeness (i.e. rare occurrence of the events), respectively. The HW PSD data can be used in the frequency range from 1 to approx. $1000 \mathrm{~Hz}$, for PIVS data the valid range is $f=(1,10) \mathrm{Hz}$, and for PIVD data $f=(10,100) \mathrm{Hz}$.

Table 2. Spectral frequency limit.

\begin{tabular}{c|ccc}
\hline & $\boldsymbol{H} \boldsymbol{W}$ & $\boldsymbol{P I V D}$ & $\boldsymbol{P I V S}$ \\
\hline$f_{N Y}[\mathrm{~Hz}]$ & 12500 & 500 & 50 \\
$f_{S R}[\mathrm{~Hz}]$ & $U / 0.0006$ & $U / 0.0018$ & $U / 0.0018$ \\
$f_{S T}[\mathrm{~Hz}]$ & $1 / 40$ & $1 / 4$ & $1 / 40$ \\
\hline
\end{tabular}

There is a wide spread of PSD curves at the height $z=0.33 \delta$ for both measurement methods, all setups, and all free stream velocities when plotted together (Fig. 4, left). The normalised energy spectra (also called premultiplied spectra) are usually plotted as $f S_{u u} / u^{* 2}$, where the energy is frequency weighted and normalised by the friction velocity squared. The frequency is normalised by the Taylor's hypothesis assumption that the structure size $\lambda$ and its frequency $f$ is linked by the local wind speed $U$, according to the formula $f=U / \lambda$. The frequency is normalised by the characteristic frequency $f_{z}=U / z$, where $z$ is wall-normal distance, leading to dimensionless frequency $n=f z / U$. Dimensionless spectral curves falls in a compact ensemble at the given height and follows $-5 / 3$ slope within the inertial sublayer (Fig. 4 , right).

The frequencies can be transformed to the wavelength $\lambda$ using the Taylor's hypothesis on frozen turbulence [5]

$$
\frac{\lambda}{\delta}=\frac{U}{f \delta} .
$$




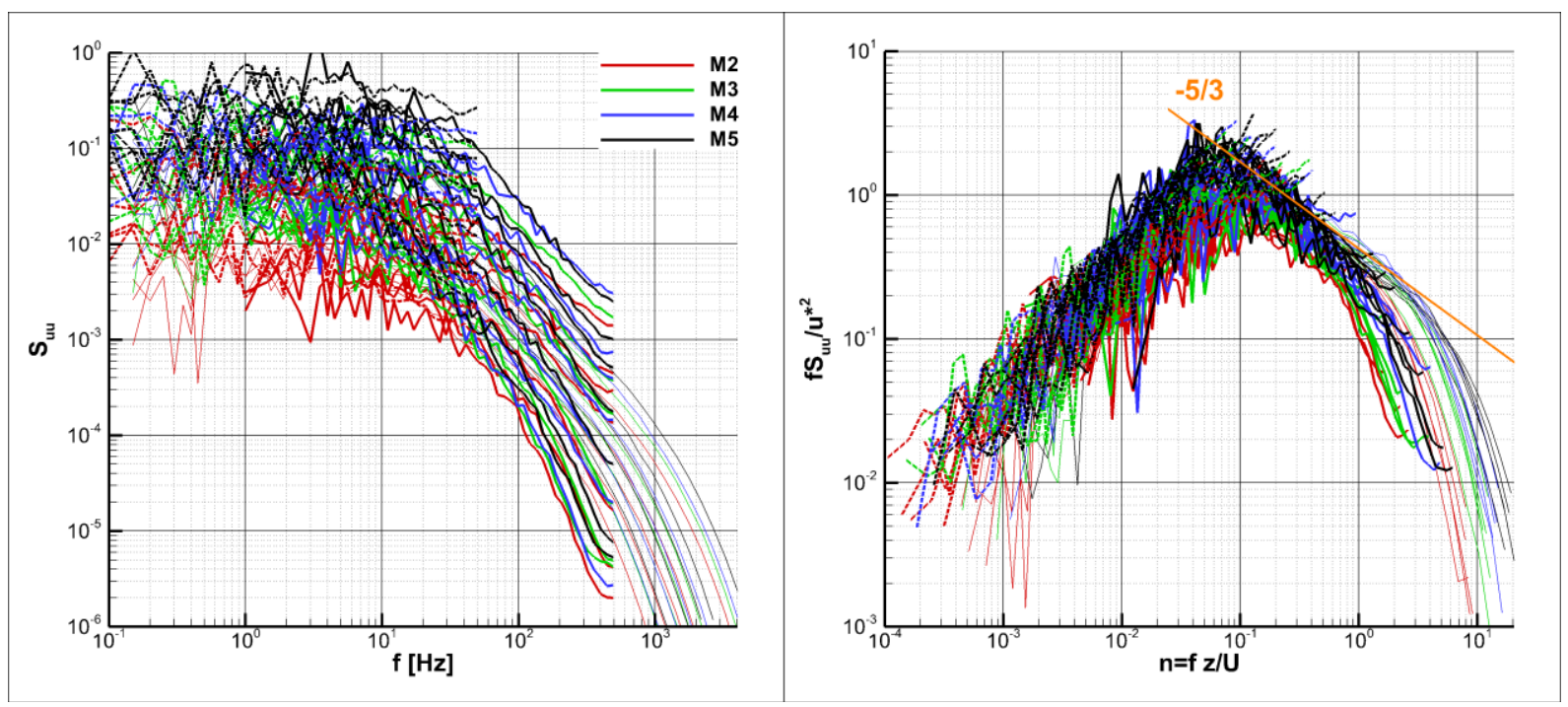

Fig. 4. Spectral curves for all setups, all free stream velocities, and both measurement method (HW thin solid line, PIVD bold solid line, PIVS dashed line) at the height $z=0.33 \delta$ in dimensional representation (left) and dimensionless form (right).

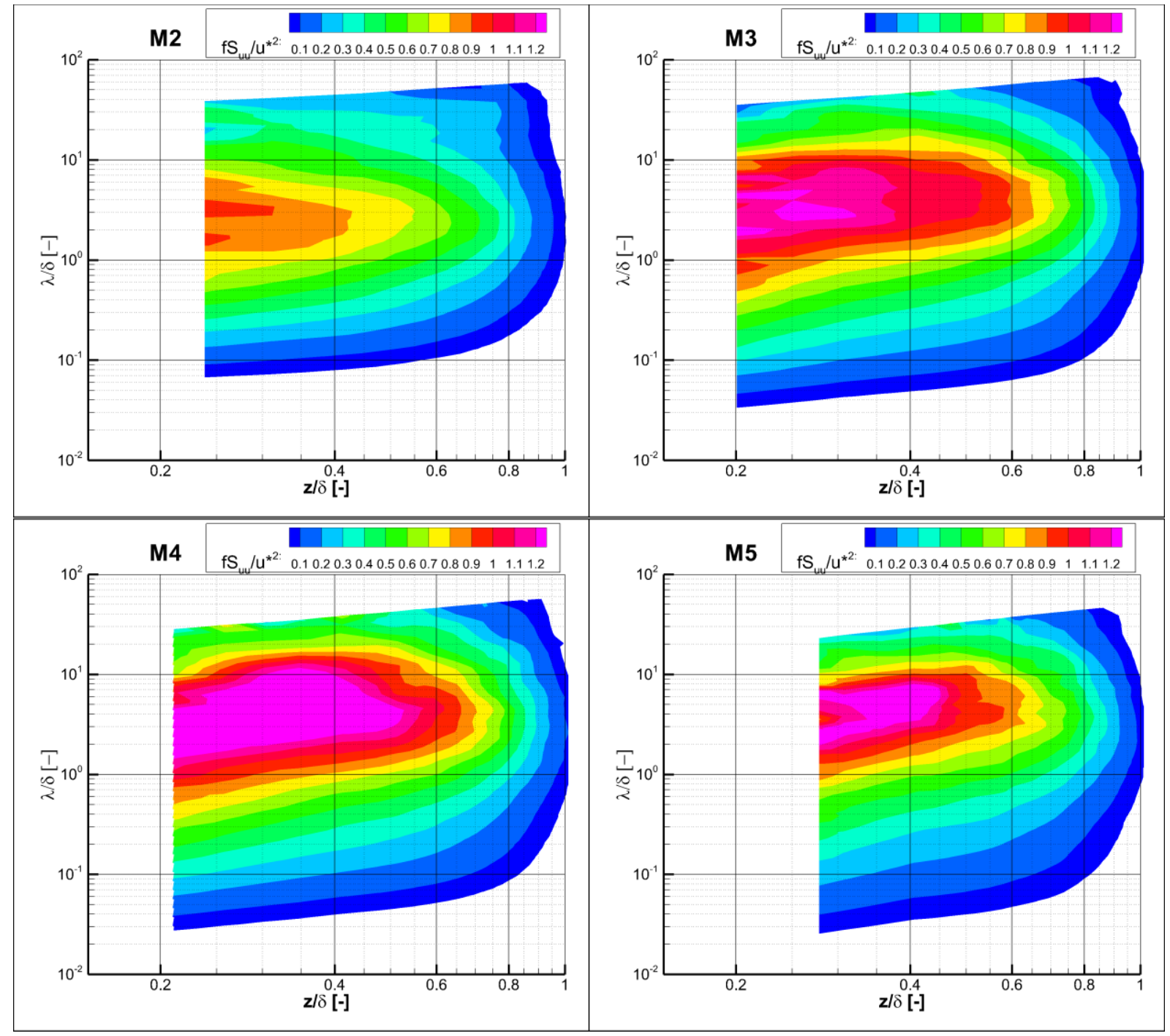

Fig. 5. Spectrograms for all setups based on the HW data. M2 $R e_{\tau}=1300, \mathrm{M} 3 R e_{\tau}=3110, \mathrm{M} 4 R e_{\tau}=4270, \mathrm{M} 5 e_{\tau}=5480$. 
Spectrograms in Fig. 5 show the premultiplied spectra as a function of the wall-normal distance $z / \delta$ (abscissa) and the wavelength $\lambda / \delta$ (ordinate). The wavelength of the most energetic structures is increasing with the wall distance, which is in agreement with the attached eddy hypothesis proposed by Townsend [6]. The actual wavelength is about $3 \delta$ at the lower elevations, while it is approaching $8 \delta$ for the upper elevations of M4 and M5 setups.

There were only minor differences in the investigated $R e_{\tau}$ ranges for each setup. The range of investigated free stream velocities was the same for all setups, however the ranges of investigated $R e_{\tau}$ were different due to different $u * / U_{\delta}$ ratios. Therefore the direct comparison between different roughness is not possible, because the differences caused by the different roughness and different Reynolds number can't be separated in this dataset.

\section{Summary and outlook}

The characteristics of the boundary layers depend on the surface roughness. The particle image velocimetry and the $x$-probe thermal anemometry showed a good agreement in the results within the method's limitations. The energetic spectra showed $-5 / 3$ slope in the inertial layer and the most energetic structures had wavelength from $3 \delta$ to $8 \delta$ depending on both the surface roughness and wall-normal distance. The spectral characteristics in wide frequency range were directly measured by HW and satisfactory reconstructed by PIVS and PIVD experiments. An upcoming measurement campaign will focus on widening and unifying $R e_{\tau}$ ranges for direct comparison of different setups.

This work was carried out thanks to support of the Czech Science Foundation (projects no. GA18-09539S and GA1518964S) and the institutional support RVO 61388998.

\section{References}

1. Schlichting, H. Boundary-Layer Theory, 7th ed., McGraw Hill, New York, U.S.A. (1979)

2. Perry, A. E. H, Enbest, S. \& Chong, M. S., J. Fluid Mech. 165, 163-199, (1986)

3. Jimenez, J., Annu. Rev. Fluid Mech. 36, 173-196, (2004)

4. Castro, I. P., J. Fluid Mech. 585, 469-485, (2007)

5. Taylor, G. I. Proc. R. Soc. Lond. A 164, 476-490, (1938)

6. Townsend, A. A. The Structure of Turbulent Shear Flow, 2nd edn. Cambridge University Press, (1976) 